\title{
Electron spectroscopic investigation of metal-insulator transition in $\mathrm{Ce}_{1-x} \mathrm{Sr}_{x} \mathrm{TiO}_{3}{ }^{\mathrm{TI}}$
}

\author{
U MANJU $^{1}$, S R KRISHNAKUMAR ${ }^{1,2}$, SUGATA RAY $^{1}$, S RAJ $^{1}$, \\ M ONODA $^{3}$, C CARBONE $^{4}$ and D D SARMA ${ }^{1, *+}$ \\ ${ }^{1}$ Solid State and Structural Chemistry Unit, Indian Institute of Science, \\ Bangalore 560 012, India \\ ${ }^{2}$ International Centre for Theoretical Physics (ICTP), Strada Costiera 11, \\ 34100 Trieste, Italy \\ ${ }^{3}$ Institute of Physics, University of Tsukuba, Tennodai, Tsukuba, 305-8571, \\ Japan \\ ${ }^{4}$ ISM-CNR sede distaccata Trieste S. S. 14, Km. 163.5 I-34012 Basovizza \\ (TS), Italy \\ ${ }^{+}$Also at Jawaharlal Nehru Centre for Advanced Scientific Research, \\ Bangalore 560 064, India \\ e-mail: sarma@sscu.iisc.ernet.in
}

\begin{abstract}
We have carried out detailed electron spectroscopic investigation of $\mathrm{Ce}_{1-}$ ${ }_{x} \mathrm{Sr}_{x} \mathrm{TiO}_{3}$ exhibiting insulator-metal transition with $x$. Core level X-ray photoelectron spectra of Ce $3 d$ as well as resonant photoemission spectra obtained at the Ce $4 d \rightarrow 4 f$ resonant absorption threshold establish $\mathrm{Ce}$ as being in the trivalent state throughout the series. Using the 'off-resonance' condition for Ce $4 f$ states, we obtain the Ti $3 d$ dominated spectral features close to $E_{F}$, exhibiting clear signatures of coherent and incoherent peaks. We discuss the implications of our findings in relation to the metalinsulator transition observed in this series of compounds.
\end{abstract}

Keywords. Photoemission; metal-insulator transition; $\mathrm{Ce}_{1-x} \mathrm{Sr}_{x} \mathrm{TiO}_{3}$; strong correlation.

\section{Introduction}

Electronic structure of transition metal (TM) oxides has been under detailed investigations in the last few years as they exhibit a wide spectrum of properties like metalinsulator transitions, colossal magnetoresistance and high temperature superconductivity. However, it has proven to be difficult to obtain a complete understanding of such phenomena, primarily due to the presence of strong electron-electron interactions within the transition element $3 d$ states. Indeed, it is the competition between the localising effects of such interactions and the comparable hopping strengths driving the system towards delocalisation, that is responsible for the wide spectrum of interesting and often exotic properties in these systems. It is important to realise that besides the Coulomb interaction and hopping strengths, there are several other electronic interactions of comparable strengths, such as the crystal field, orbital angular momentum dependence of Coulomb interactions essentially connected with the orbital degeneracy, that influence

\footnotetext{
IIDedicated to Professor C N R Rao on his 70th birthday

*For correspondence
} 
the electronic structure. In this respect it is most profitable to investigate transition metal systems in the low electron-filling limit near the $d^{1}$ configuration. In particular, there is a large number of interesting transition metal compounds with the electron occupancy varying from 0 and $1 .^{1,2}$ On one end of the spectrum, the $d^{0}$ system is a band insulator and on the other end, a $d^{1}$ system has strong electron-electron interaction effects. Thus, by tuning the electron occupancy per site, one can study the evolution of electron correlations in the electronic structure of such compounds.

Photoemission spectroscopy of early TM oxides with $d^{1}$ electronic configuration reveal $^{3,4}$ that there are two spectral features related to the transition metal $3 d$ states, namely the coherent feature at the Fermi level $\left(E_{F}\right)$ and the incoherent feature away from $E_{F}$. It is known that the intensity ratio of the coherent and the incoherent spectral features changes systematically as a function of $U / W$, where $U$ is the on-site Coulomb interaction energy and $\mathrm{W}$ is the band width. This is consistent with theoretical predictions based on the single band Hubbard model. ${ }^{5}$ A particularly interesting system to investigate in this context is $\mathrm{Ce}_{1-x} \mathrm{Sr}_{x} \mathrm{TiO}_{3}$, which so far has been sparingly investigated within high energy spectroscopic techniques.

$\mathrm{CeTiO}_{3}$ is a Mott-Hubbard insulator with an on site $U$ of about $2-3 \mathrm{eV}^{6-8}$ at the Ti site. Here $\mathrm{Ce}$ is believed to be in the $\mathrm{Ce}^{3+}$ state making the compound a $d^{1}$ system. $\mathrm{SrTiO}_{3}$ is a $d^{0}$ band insulator. Since $\mathrm{Sr}$ content $(x)$ in $\mathrm{Ce}_{1-x} \mathrm{Sr}_{x} \mathrm{TiO}_{3}$ can be varied over a wide composition range the system can be doped continuously ${ }^{9}$ varying the average electron occupancy of the $3 d$ states between 0 and 1 . On substituting Ce with $\mathrm{Sr}, \mathrm{Ce}_{1-x} \mathrm{Sr}_{x} \mathrm{TiO}_{3}$ exhibits an insulator to metal transition for small values of $x(\approx 0 \cdot 05) .^{9}$

\section{Experiments}

Polycrystalline samples of $\mathrm{Ce}_{1-x} \mathrm{Sr}_{x} \mathrm{TiO}_{3}$, with $x=0,0.1,0 \cdot 2,0.3,0.4$ and 0.7 were prepared by inert gas (Ar) melting. ${ }^{9}$ The starting materials used were $\mathrm{CeO}_{2}(99.9 \%$ purity), $\mathrm{SrO}$ (99\% purity), $\mathrm{TiO}_{2}(99.9 \%$ purity) and $\mathrm{Ti}$ metal (99.9\% purity). All samples were characterized by X-ray diffraction measurements and found to be in single phase without any detectable impurities. Ultraviolet photoemission experiments were performed on these samples at room temperature. In order to get high quality electron spectroscopic information from these samples, we investigated the core level regions using monochromatized AlK $\alpha$ radiation in a custom-built multi-technique electron spectrometer manufactured by VSW, UK. The base pressure in this case was $5 \times 10^{-10}$ mbar. The valence band investigation was carried out at the Vacuum Ultraviolet (VUV) photoemission beamline, $3 \cdot 2 \mathrm{R}$ at the Italian synchrotron radiation centre, Elettra, Trieste. The base pressure in this case was $1 \times 10^{-9}$ mbar. The sample surface was cleaned in situ by repeated scraping with a diamond file and the cleanliness was monitored by collecting the $\mathrm{O} 1 s$ and $\mathrm{C} 1 s$ spectral regions. In every case, $\mathrm{C} 1 s$ signal was found to be essentially below the level of detection, whereas the $\mathrm{O} 1 s$ spectra presented a single peak structure without evidence of any impurity feature.

\section{Results and discussion}

Ce in $\mathrm{Ce}_{1-x} \mathrm{Sr}_{x} \mathrm{TiO}_{3}$ is expected to be in a trivalent state, considering its coordination number and $\mathrm{Ce}-\mathrm{O}$ bond distances, for all $x$. This implies that $\mathrm{Ti}$ is in $\mathrm{Ti}^{3+}$ state in $\mathrm{CeTiO}_{3}$, continuously evolving towards $\mathrm{Ti}^{4+}$ state as the $\mathrm{Sr}$ content, $x$, approaches unity. However, any transformation, even a partial one, to tetravalent $\mathrm{Ce}$ will correspondingly change the 
$3 d$ electronic configuration of the system. Therefore, it is necessary to determine the actual valence state of $\mathrm{Ce}$ in these compounds. For this purpose, we have recorded the $\mathrm{Ce}$ $3 d$ core level signal from several compositions in this series, as Ce $3 d$ core level spectra are known ${ }^{10}$ to bear characteristic and distinct features of $\mathrm{Ce}^{3+}$ and $\mathrm{Ce}^{4+}$ states in corresponding compounds. We show a selection of Ce $3 d$ spectra for various values of $x$ in figure 1; the spectra appear to be very similar to one another. The spectral features in the energy range of $880-890 \mathrm{eV}$ correspond to $\mathrm{Ce} 3 d_{5 / 2}$ signal, while those in the energy range of $900-910 \mathrm{eV}$ correspond to the $\mathrm{Ce} 3 d_{3 / 2}$ signal. Each of these two regions shows clearly the evidence of a doublet feature, namely a main peak and a satellite feature approximately separated by $4.3 \mathrm{eV}$ arising from many-electron processes leading to different final states following the photoionization. Comparing with the published results ${ }^{11,12}$ of $\mathrm{Ce}_{2} \mathrm{O}_{3}$ and $\mathrm{CeO}_{2}$, it becomes evident that $\mathrm{Ce}$ is in the trivalent state in all these cases.

We have recorded the valence band photoemission spectra of these samples with a wide range of incident photon energies. As a typical example, we show a selection of spectra obtained from $\mathrm{Ce}_{0.9} \mathrm{Sr}_{0.1} \mathrm{TiO}_{3}$ with the photon energy varying from $25 \mathrm{eV}$ to $125 \mathrm{eV}$ in the main panel of figure 2 . We can clearly see a broad and intense feature around $4-9 \mathrm{eV}$ binding energy; this feature, common to all oxide samples, ${ }^{10,13}$ arises from the band states with a predominant oxygen $2 p$ character. While the plots in figure 2 are

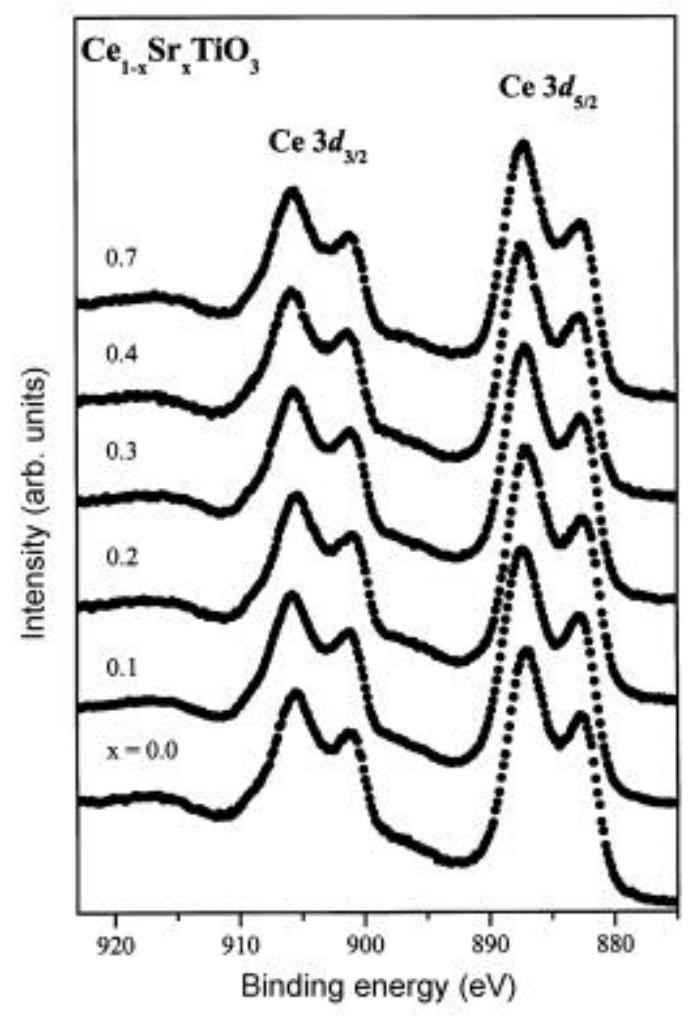

Figure 1. Ce $3 d$ core level photoemission spectra from $\mathrm{Ce}_{1-x} \mathrm{Sr}_{x} \mathrm{TiO}_{3}$ for various values of $x$. 


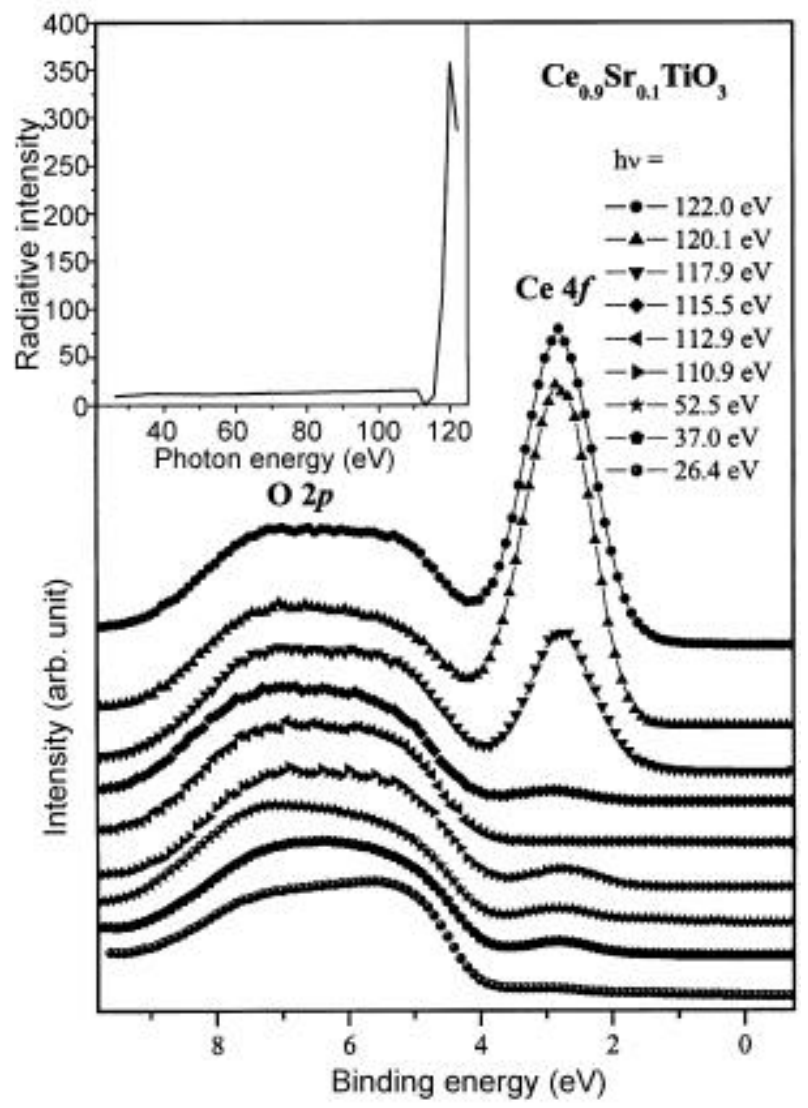

Figure 2. Valence band spectra of $\mathrm{Ce}_{0.9} \mathrm{Sr}_{0.1} \mathrm{TiO}_{3}$ recorded with various incident photon energies. The inset shows the relative intensity variation of Ce $4 f$ peak intensity with respect to $\mathrm{O} 2 p$ as a function of photon energy.

made by normalizing the spectral intensity at this feature, we have observed a monotonic decrease in the intensity of this feature with increasing photon energies, consistent with the known reduction of oxygen $2 p$ cross-section with photon energy. ${ }^{14}$ However, the most remarkable intensity variation is observed for the spectral feature at about $3 \mathrm{eV}$ binding energy. This feature initially gains in intensity monotonically as the incident photon energy $(h \mathbf{v})$ is increased from 26 to $111 \mathrm{eV}$. After that with a small increase in the incident photon energy to around $112 \mathrm{eV}$, this feature almost completely vanishes. Again as the photon energy is increased, the intensity of this feature increases sharply reaching a maximum at about $h \mathrm{v}=122 \mathrm{eV}$. We have plotted the intensity variation of this feature relative to the $\mathrm{O} 2 p$ feature at $5 \cdot 3 \mathrm{eV}$ as a function of the incident photon energy in the inset to figure 2 . This plot with very rapid changes in the cross section in the photon energy range of $110-130 \mathrm{eV}$ clearly identifies this feature as arising from Ce $4 f$ states. This rapid oscillation in the cross-section of this feature for $h \mathbf{v}=110-130 \mathrm{eV}$ is due to the resonant photoemission process involving the Ce $4 d \rightarrow 4 f$ resonant excitation. ${ }^{15}$ At $h v=122 \mathrm{eV}$, the 'on-resonance' condition of Ce $4 d$ to $4 f$ excitation ${ }^{15}$ enhances the Ce $4 f$ related intensity manifold, while $h \mathrm{v}=112 \mathrm{eV}$ represents the so-called 'off-resonance' 
condition where the cross-section for the Ce $4 f$ states is drastically reduced. It is to be noted that such a moderate change of $h \mathrm{v}$ from $112 \mathrm{eV}$ to $122 \mathrm{eV}$ leaves the cross-sections of all the other states essentially unchanged. This allows us to construct a 'difference' spectrum, where we subtract out the spectrum recorded with $h \mathrm{v}=112 \mathrm{eV}$ from the spectrum recorded with $h \mathrm{v}=122 \mathrm{eV}$. This difference spectrum, with all contributions from states other than the $\mathrm{Ce} 4 f$ removed by the process of subtraction, represents the $\mathrm{Ce}$ $4 f$ spectrum and is shown for $\mathrm{Ce}_{0.9} \mathrm{Sr}_{0 \cdot 1} \mathrm{TiO}_{3}$ in figure 3. It clearly shows the Ce $4 f$ peak at $2 \cdot 8 \mathrm{eV}$ with no intensity at $E_{F}$. This clearly suggests that Ce is in an essentially pure $\mathrm{Ce}^{3+}$ state in this compound, consistent with conclusions drawn from Ce $3 d$ core level spectra shown in figure 1.

We have carried out similar experiments on various samples in this series. We show a selection of spectra obtained from $\mathrm{Ce}_{0.7} \mathrm{Sr}_{0.3} \mathrm{TiO}_{3}$ recorded with the photon energy ranging between $25 \mathrm{eV}$ to $122 \mathrm{eV}$ in figure 4 . Evidently, the spectral features show changes similar to the ones exhibited by $\mathrm{Ce}_{0.9} \mathrm{Sr}_{0.1} \mathrm{TiO}_{3}$ (see figure 2). Thus, we find a nearcomplete absence of $\mathrm{Ce} 4 f$ related signal at about $3 \mathrm{eV}$ binding energy in the spectrum recorded with $h \mathrm{v}=112 \mathrm{eV}$, while the Ce $4 f$ intensity appears to reach a maximum with $h v=122 \mathrm{eV}$. Once again a difference spectrum (not shown here) establishes as essentially pure $\mathrm{Ce}^{3+}$ state of $\mathrm{Ce}$ in this compound also.

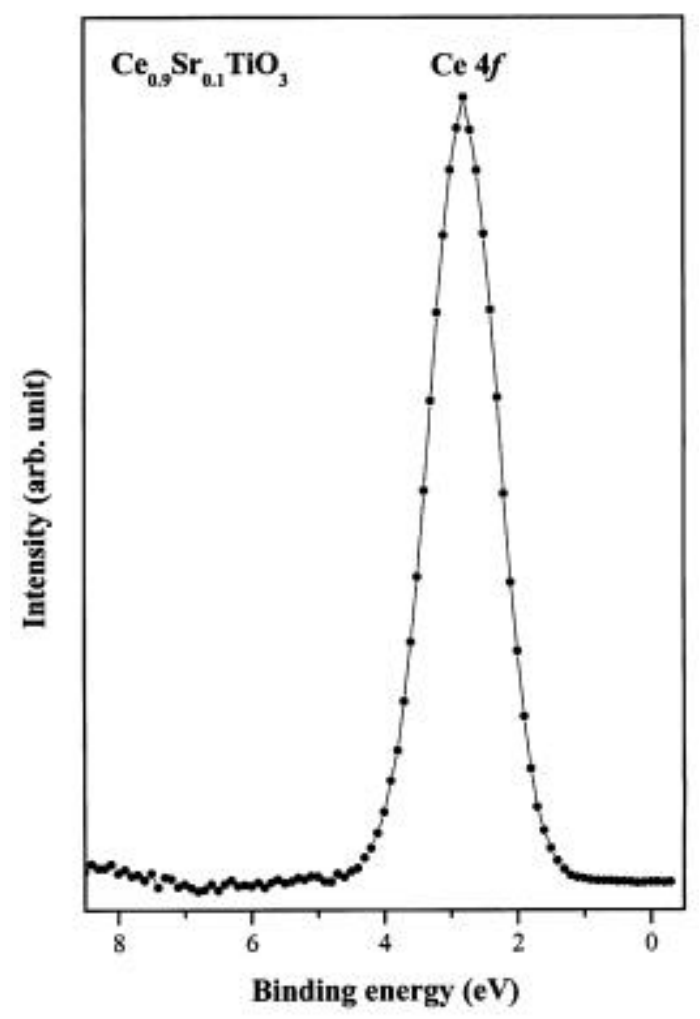

Figure 3. Difference spectrum constructed from the spectra recorded with photon energies corresponding to Ce $4 f$ on-resonance and off-resonance conditions, representing Ce $4 f$ spectral features. 


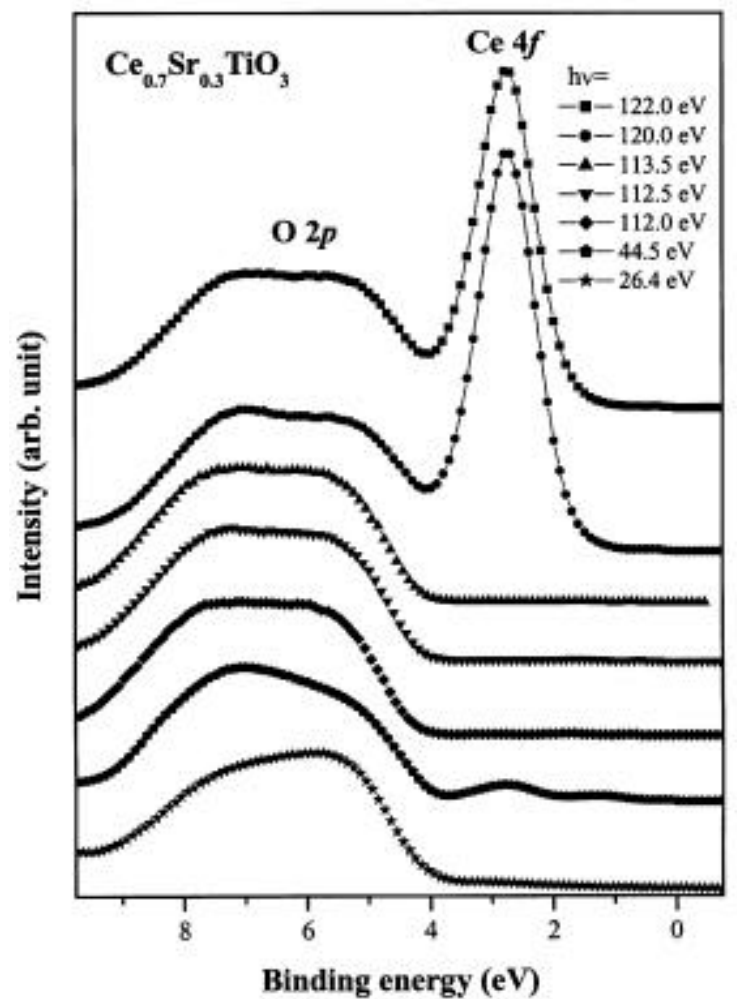

Figure 4. Valence band spectra of $\mathrm{Ce}_{0.7} \mathrm{Sr}_{0.3} \mathrm{TiO}_{3}$ recorded with various incident photon energies.

As $\mathrm{Ce}$ is in the trivalent state, the electronic properties of this series of compounds is dominated by the Ti $3 d$ states hybridised with oxygen $2 p$ states. In the metallic samples, these states ought to give rise to finite intensities at the Fermi energy, while the insulating-end compounds are expected to show the signature of a finite gap at $E_{F}$. However, the strong $\mathrm{Ce} 4 f$ peak and its resolution broadened extensions towards the lower binding energy side completely mask the other features near $E_{F}$. In order to study the states near $E_{F}$ responsible for the metal-insulator transition in these compounds, we make use of the 'off-resonance' condition at $h \mathrm{~V}=112 \mathrm{eV}$, so that the Ce $4 f$ intensity is all but completely suppressed from the spectra. This allows us to record the low intensity $\mathrm{Ti}$ $3 d$ related spectral features. Figure 5 shows the valence band spectra near $E_{F}$ recorded with $h v=112 \mathrm{eV}$ for various samples. The spectrum of $\mathrm{CeTiO}_{3}$ has a single spectral feature with a peak around $1.2 \mathrm{eV}$ binding energy; we find that the nearly-negligible spectral intensity at $E_{F}$ is accounted for by the resolution broadening of the feature at $1.2 \mathrm{eV}$. This is the spectral signature of the lower Hubbard band arising from $d^{1} \rightarrow d^{0}$ transition and corresponds to the electrons that are localised due to strong electron correlation effects. Consequently, there is no intensity at $E_{F}$, consistent with the insulating nature of the compound. With increasing level of doping of $\mathrm{Sr}$ for $\mathrm{Ce}$, two changes are found in the spectral region. First of all, the intensity of the spectral signature of the lower Hubbard band decreases steadily with increasing level of doping; this missing intensity from the lower Hubbard band reappears as a sharp spectral feature at 
$E_{F}$. This feature exhibits increasing intensity as a function of doping, $x$. It is evident that this feature has a substantial intensity at $E_{F}$; moreover, the decrease of the spectral intensity near and above $E_{F}$ is clearly related to the Fermi-Dirac statistics. Therefore, it is clear that this feature represents the delocalised electrons in the systems consistent with the metallic state at these doping levels; this spectral feature is termed ${ }^{3}$ as the coherent feature.

It is interesting to note that even for the samples deep in the metallic regime such as, $\mathrm{Ce}_{0.7} \mathrm{Sr}_{0.3} \mathrm{TiO}_{3}$, the incoherent feature continues to be more intense than the coherent feature. This is in sharp contrast with theoretical results ${ }^{5}$ that predict rapid loss of intensity for the spectral signature of the lower Hubbard band or the incoherent spectral feature with doping. Similar results have been reported also for other early transition metal oxide systems. ${ }^{16-18}$ A substantial part of this disagreement is clearly due to a strong difference in the surface and the bulk electronic structures of such compounds. Similar differences between the surface and the bulk electronic structures have already been established for other transition metal oxides like $\mathrm{La}_{1-x} \mathrm{Ca}_{x} \mathrm{VO}_{3}{ }^{16,19}$ and $\mathrm{Ca}_{1-x} \mathrm{Sr}_{x} \mathrm{VO}_{3}{ }^{4}$; similar effects have also been reported compounds of cerium. ${ }^{20,21}$

\section{Conclusions}

We have reported core level $\mathrm{Ce} 3 d$ spectra from $\mathrm{Ce}_{1-x} \mathrm{Sr}_{x} \mathrm{TiO}_{3}$ samples to establish a trivalent state of $\mathrm{Ce}$ in these compounds for all values of $x$. We also carried out extensive VUV photoemission experiments on these samples with the photon energy varying between $25-125 \mathrm{eV}$. Difference spectrum obtained by subtracting the off-resonance spectrum from the on-resonance one, we obtain the Ce $4 f$ spectral signature; thus

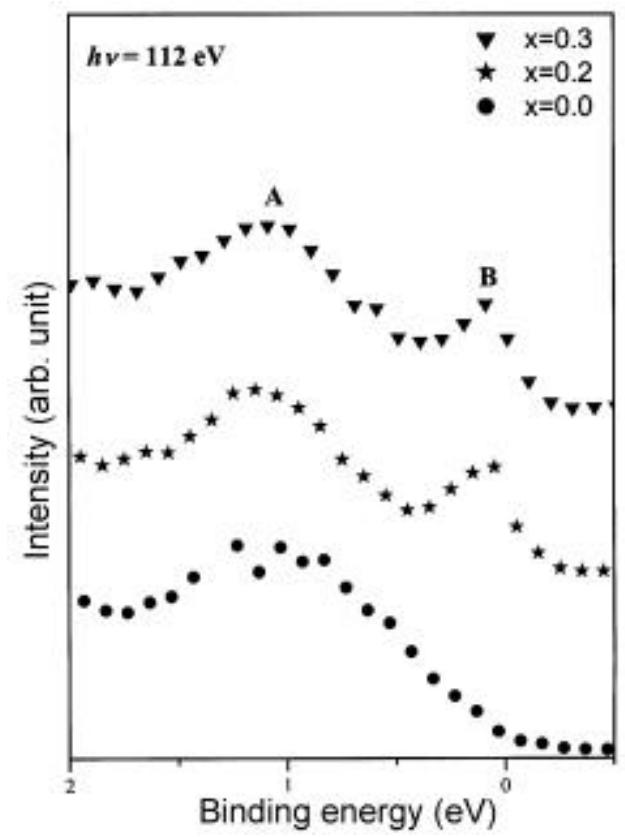

Figure 5. Valence band spectral features near $E_{F}$ for $\mathrm{Ce}_{1-x} \mathrm{Sr}_{x} \mathrm{TiO}_{3}$ with $x=0 \cdot 0,0 \cdot 2$ and $0 \cdot 3$, measured with $112 \mathrm{eV}$ photon energy, showing the metal-insulator transition as a function of $x$. 
obtained Ce $4 f$ spectrum which has a peak at about $3 \mathrm{eV}$ binding energy and shows no intensity at $E_{F}$ even for the metallic samples, consistent with a $\mathrm{Ce}^{3+}$ state. By recording the valence band spectra at the Ce $4 f$ off-resonance condition, the coherent and the incoherent spectral features arising from the $\mathrm{Ti} 3 d$ states could be clearly resolved, allowing us to investigate the metal insulator transition in the $\mathrm{Ce}_{1-x} \mathrm{Sr}_{x} \mathrm{TiO}_{3}$ system as a function of $\mathrm{Sr}$ or hole doping. The experimental spectra of the metallic compounds exhibit an intensity of the incoherent feature considerably larger than that predicted by theory. This discrepancy is possibly due to a difference in the surface and the bulk electronic structures of these compounds.

\section{Acknowledgements}

We thank the board of Research in Nuclear Sciences and Department of Science and Technology, Government of India for financial support. MU, SR, SR and DDS gratefully acknowledge the support of the International Centre for Theoretical Physics under the ICTP-Elettra users program for synchrotron radiation. MU thanks the Council of Scientific and Industrial Research, New Delhi for a fellowship. SRK thankfully acknowledges the support of ICTP Programme for Training and Research in Italian Laboratories, Trieste, Italy.

\section{References}

1. Sarma D D, Barman S R, Kajueter H and Kotlier G 1996 Europhys. Lett. 36307

2. Fujimori A, Hase I, Nakamura M, Namatame H, Fujishima Y, Tokura Y, Abbate M, de Groot F M F, Czyzyk M T, Fuggle J C, Strebel O, Lopez F, Domke M and Kaindl G 1992 Phys. Rev. B46 9841

3. Fujimori A, Hase I, Namatame H, Fujishima Y, Tokura Y, Eisaki H, Uchida H, Takegahara K and de Groot F M F 1992 Phys. Rev. Lett. 691796

4. Maiti K, Sarma D D, Rozenberg M J, Inoue I H, Makino H, Goto O, Pedio M and Cimino R 2001 Europhys. Lett. 55246

5. Georges A, Kotlier G, Krauth W and Rozenberg M J 1996 Rev. Mod. Phys. 6813

6. Akaki O, Chainani A, Yokoya T, Fujisawa H, Takahashi T and Onoda M 1997 Phys. Rev. B56 12050

7. Reedyk M, Crandles D A, Cardona M, Garrett J D and Greedan J E 1997 Phys. Rev. B55 1442

8. Crandles D A, Timusk T, Garrett J D and Greedan J E 1992 Physica C201 407

9. Onoda M and Yasumoto M 1997 J. Phys., Condens. Matter 95623

10. Sarma D D and Rao C N R 1980 J. Electron Spectrosc. Relat. Phenom. 2025

11. Sarma D D, Hegde M S and Rao C N R 1981 J. Chem. Soc., Faraday Trans. 771509

12. Kotani A and Ogasawara H 1992 J. Electron Spectrosc. Relat. Phenom. 60257

13. Rao C N R, Sarma D D, Vasudevan S and Hegde M S 1979 Proc. R. Soc. (London)A367 239

14. Yeh J J and Lindau I 1985 At. Data Nucl. Data Tables 321

15. Lenth W, Lutz F, Barth J, Kalkoffen G and Kunz C 1978 Phys. Rev. Lett. 411185

16. Maiti K, Mahadevan P and Sarma D D 1998 Phys. Rev. Lett. 802885

17. Maiti K 1998 Novel electronic structures in transition metal oxides, $\mathrm{Ph} \mathrm{D}$ thesis, Solid State and Structural Chemistry Unit, Indian Institute of Science, Bangalore

18. Morikawa K, Mizokawa T, Fujimori A, Taguchi Y and Tokura Y 1998 Phys. Rev. B54 8446

19. Maiti K and Sarma D D 2000 Phys. Rev. B61 2525

20. Weschke E, Laubschat C, Simmons T, Domke M, Strebel O and Kaindl G 1991 Phys. Rev. B44 8304

21. Laubschat C, Weschke E, Holtz C, Domke M, Strebel O and Kaindl G 1990 Phys. Rev. Lett. 651639 